\title{
Aftershock sequence simulations using synthetic earthquakes and rate-state seismicity formulation
}

\author{
Heming Xu • Yifeng Cui • James H. Dieterich • \\ Keith Richards-Dinger · Efecan Poyraz • \\ Dong Ju Choi
}

Received: 27 November 2013/ Accepted: 7 May 2014/Published online: 11 July 2014

(C) The Seismological Society of China, Institute of Geophysics, China Earthquake Administration and Springer-Verlag Berlin Heidelberg 2014

\begin{abstract}
We use an efficient earthquake simulator that incorporates rate-state constitutive properties and uses boundary element method to discretize the fault surfaces, to generate the synthetic earthquakes in the fault system. Rate-and-state seismicity equation is subsequently employed to calculate the seismicity rate in a region of interest using the Coulomb stress transfer from the main shocks in the fault system. The Coulomb stress transfer is obtained by resolving the induced stresses due to the fault patch slips onto the optimal-oriented fault planes. The example results show that immediately after a main shock the aftershocks are concentrated in the vicinity of the rupture area due to positive stress transfers and then disperse away into the surrounding region toward the background rate distribution. The number of aftershocks near the rupture region is found to decay with time as Omori aftershock decay law predicts. The example results demonstrate that the rate-and-state fault system earthquake
\end{abstract}

\author{
H. Xu $(\bowtie) \cdot$ Y. Cui · E. Poyraz · D. J. Choi \\ San Diego Supercomputer Center, University of California, \\ San Diego, CA, USA \\ e-mail: h1xu@ucsd.edu \\ Y. Cui \\ e-mail: ycui@sdsc.edu \\ E. Poyraz \\ e-mail: efecanpoyraz@gmail.com \\ D. J. Choi \\ e-mail: dchoi@sdsc.edu \\ H. Xu - J. H. Dieterich - K. Richards-Dinger \\ Department of Earth Sciences, University of California, \\ Riverside, CA, USA \\ e-mail: dieterichj@ucr.edu \\ K. Richards-Dinger \\ e-mail: keith.richards-dinger@ucr.edu
}

simulator and the seismicity equations based on the ratestate friction nucleation of earthquake are well posited to characterize the aftershock distribution in regional assessments of earthquake probabilities.

Keywords Earthquake simulator - Rate-and-state seismicity · Aftershock

\section{Introduction}

Earthquakes occur in response to continuous tectonic forcing and from interactions among elements of fault networks that slip and transfer stresses. Following a large earthquake occurrence of aftershocks in time and space is related to that main shock. Those aftershocks could last for days or years and pose as seismic hazard after a main shock. Aftershock sequences or earthquake interactions, such as earthquake triggering and earthquake suppressing, have been widely investigated in the context of earthquakeinduced static or dynamic stress changes. It has been shown for decades that large earthquakes can inhibit or promote failure on nearby faults in form of such stress transfer. The abundance of phenomenological investigations has shown that spatial patterns of static or dynamic stress changes seem to correlate well with spatial patterns of aftershocks for natural earthquakes (e.g. Harris 1998; Kilb et al. 2002; Steacy et al. 2005a; Durand et al. 2013). It becomes a commonplace understanding that positive stress changes tend to activate faults, triggering more failures, resulting in more aftershocks, whereas negative stress changes tend to relax faults, suppressing further failures, diminishing the possible aftershocks.

Generally, the Coulomb stress transfer due to an earthquake is calculated by summing the stress changes due to 
all slipping fault patches using elastic dislocation theory (Okada 1992) and resolving the resulting stresses onto the plane of interest such as pre-existing fault planes or optimally oriented planes (OOP) determined by stress states. The OOP are strongly dependent on the orientation of the regional stress field and can vary widely in an active seismic region. The predictive stress transfer map might look quite different depending on assumption about the orientation of the active structures. The optimal planes could be determined from a combination of regional stress field and main shock-induced stress field using the maximum possible Coulomb failure plane orientation. This method shows that distribution of aftershocks of natural earthquakes can be explained by the Coulomb criterion (e.g. King et al. 1994; Toda et al. 2005). The optimal planes might be computed in a 3D stress field with some constraints placed on the fault orientation (McCloskey et al. 2003; Steacy et al. 2005b; Xu et al. 2010). Such a method uses the available geological structure information in a region of interest and fixes one of the fault orientations. The predictions by such a method would fit the spatial distribution of the events better than using unconstrained fault orientation in case studies (e.g. McCloskey et al. 2003; Steacy et al. 2005a).

The Coulomb failure hypothesis is conceptually simple and convenient to implement. It has been used for decades to explain the characteristics of aftershocks, however, it does have limitations. In the investigation of suppression of large earthquakes, Harris and Simpson (1998) found that a new failure model, rate-state formulation, offers a consistent explanation for the aftershocks after 1906 San Francisco earthquake whereas the Coulomb failure model does not. Unlike the Coulomb failure model, the new failure model is based on rate- and state-dependent friction constitutive representation of laboratory observations, and appears more complex by describing the evolution of quantities as they approach failure limits though it was shown that rate-state models might asymptotically become equivalent to Coulomb models under a variety of conditions (Gomberg et al. 2000). It has also been used to examine the earthquake probabilities by transient rate-andstate triggering effects and used to forecast the evolution of seismicity in southern California (Toda et al. 1998, 2005) and to model the aftershock sequences with 3D stress heterogeneities and planar/rough fault models (Smith and Dieterich 2010). The rate-and-state model has been compared with the Coulomb stress model on predictions of clock advance (Gomberg et al. 1998, 2000). The rate-andstate parameters can be found by fitting aftershock sequences (Gross and Burgmann 1998; Harris and Simpson 1998). Favorably, the rate-and-state formulation explains temporal features of aftershocks, such as the Omori law decay in aftershock seismicity rate as consequence of
Coulomb stress transfers and predicts that aftershock duration is proportional to mainshock recurrence time (Dieterich 1994, 2007). Based on the rate-and-state constitutive model, the seismicity rate equation describing the evolution of seismicity rate with stress transfer could be used to determine earthquake probabilities in a region of interest and that the seismicity rate is a function of time and the stress transfer (Harris and Simpson 1998; Toda et al. 2005; Hainzl et al. 2009). Such a synthetic aftershock catalog might be obtained using the rate-state seismicity rate as a non-stationary statistical process and the catalog might serve as a regional seismic hazard estimator which depicts some characteristics of the aftershock occurrence in time and space after an event (Dieterich 1994; Smith and Dieterich 2010).

The rate-state off-fault seismicity rate is determined in term of the geophysical variables such as the stressing rate that determines the seismicity decay characteristics with time and Coulomb stress transfer that changes the seismicity rate instantaneously at earthquake occurrence. In addition to the rate-state representation model, earthquake occurrence can be described as a stochastic process. One is the stress release model (Vere-Jones 1978) based on elastic rebound theory. This model assumes the occurrence probability depends on some quantity which may be interpreted as the mean-stress level in a region and increases with an increasing stress level and drops when an earthquake happens (e.g. Keuhn et al. 2008). Stress transfer and stresstriggering between distant faults can be included to simulate realistic scenarios. Note that the stress release is connected with the earthquake magnitude empirically. The other is the epidemic-type aftershock sequence (ETAS) model (Ogata 1988). The ETAS model describes earthquake activity as a point process, and the earthquake occurrence rates are history dependent and depend on four constants that are aftershock productivity factor, power-law exponent of the event rate decay in the Omori law, magnitude sensitivity parameter, and characteristic time shift. The four model constants and the background seismic rate can be estimated from the regional aftershock seismicity observations. This model and its extensions have been shown useful for quantifying the seismic activation and quiescence in earthquake active regions, and some results suggest the background seismicity might increase with time in some regions (e.g. Ogata et al. 2003; Bansal and Ogata 2013; Kumazawa and Ogata 2013).

We investigate off-fault aftershock sequences from the simulated earthquakes in the fault system calculated by an efficient earthquake simulator (Dieterich and RichardsDinger 2010) in this article. The simulator is based on ratestate friction nucleation of earthquake and can resolve the discrete fault-slip events across the scale range needed to track the state evolution for the brittle regions of the solid 
Earth using appropriate constitutive friction law so that we could better understand the short-term dynamics of how fault-system state evolves with long-term tectonic behavior. This type of multi-scale simulation spans a continuous range of time scales from $<10^{-3}$ s for slip evolution during fault rupture to $\sim 10^{3} \mathrm{~s}$ for the inertial dynamics of largescale ruptures, to $\geq 10^{11} \mathrm{~s}$ for the characterization of longterm deformation and large-event statistics. Corresponding spatial scales extend from $\leq 100 \mu \mathrm{m}$ to describe the evolution of fault properties during slip to $>100 \mathrm{~km}$ to represent plate boundary fault systems. Such an earthquake simulator generates an earthquake sequence catalog over a long-period window and has been used in study of characteristic earthquake recurrence in a nonplanar fault system (e.g. Dieterich and Richards-Dinger 2010). The produced earthquakes (slips on the fault surface) in the fault system are then used to calculate the static stress changes off-fault in a region in a physical material model (Okada 1992). The stress transfers depend on the source/receiver locations, source mechanism and receiver fault's orientation that is determined by the stress field (e.g. King et al. 1994). The seismicity rate is calculated using the rate-state formulation and the aftershock sequence is obtained by drawing from the seismicity rate at each grid point (cell center). The background seismicity rate in this study is set to be constant and uniform. Finally, the synthetic regional aftershock catalog is obtained by merging and sorting the aftershock sequences at each grid point for further regional aftershock analysis.

\section{Methodology}

In this study, we first summarize the method to calculate the synthetic earthquake catalog in a fault system using the earthquake simulator code RSQSim (Dieterich and Richards-Dinger 2010; Richards-Dinger and Dieterich 2012). The earthquake catalog consists of earthquake times and faulting information. Then the induced seismicity rate offfault in a region is calculated using the Coulomb stress change in the rate-state seismicity equations. The seismicity rate is both time-dependent and space-dependent.

\subsection{Synthetic earthquake catalog calculation}

Fault slip is the primary mode of deformation in the brittle crust, and the friction constitutive model specifies when and where failure could occur. The slip characteristics of the fault are strongly dependent on the stressing states, fault orientation, and fault surface contact properties, etc. The model used in this study employs a rate- and statedependent formulation for sliding resistance. Laboratory observations indicate that the constitutive law governing fault slip includes dependencies on slip rate and on a state variable that evolves with slip distance and time as written as follows (Dieterich 1979; Ruina 1983):

$m=m_{0}+a \ln \frac{\dot{\delta}}{\dot{\delta}^{*}}+b \ln \frac{\theta}{\theta^{*}}$,

where $m$ is the dynamic coefficient of friction, $m_{0}$ is the static coefficient of friction, $\dot{\delta}$ is slip displacement, $a$ and $b$ are experimentally determined constants, $\theta$ is a state variable that evolves with time, slip and normal stress history, dotted terms denote time-derivative, asterisked terms denote normalizing constants. This rate-and-state constitutive law provides a well-tested and integrated theoretical framework for deterministic modeling of fault behaviors ranging from fractions of a second during earthquake rupture to interseismic periods of thousands of years. In the rate-and-state formalism, earthquakes nucleate at some elements where the sliding speed is large and the stress exceeds the steady-state friction, depending on the stressing conditions, and increases rapidly above interseismic values. The cycle of stress accumulation and earthquake slip at each fault element loops among three states (Dieterich 1995; Ziv and Rubin 2003): state 0 for stress below the steady-state friction; state 1 for stress exceeding the steady-state friction with a stable (interseismic) slip; state 2 for unstable slip that increases dramatically above interseismic values.

The stress state change due to a fault slip is represented in term of the interaction matrix (Dieterich and RichardsDinger 2010; Richards-Dinger and Dieterich 2012):

$$
\begin{gathered}
\tau_{i}=K_{i j}^{\tau} \delta_{j}+\tau_{i}^{\mathrm{tect}}, \\
\sigma_{i}=K_{i j}^{\sigma} \delta_{j}+\sigma_{i}^{\mathrm{tect}}
\end{gathered}
$$

where $i$ indicates the index number of receiver fault element, $j$ indicates the index number of the slipped fault element, $\tau$ and $\sigma$ are the shear stress in the slip direction and fault-normal stress on the fault element, respectively, the two $k_{i j}$ for shear and normal stresses are interaction matrices derived from elastic dislocation solution at element $i$ due to a slip at element $j, \delta$ is slip on element $j$, the superscript "tect" indicates the stresses applied to the $i$ th element by sources external to the fault system (such as far field tectonic motions). The summation convention applied to repeated indices.

Based on the rate-and-state formulation the rupture model, the earthquake simulator RSQSim (Dieterich and Richards-Dinger 2010; Richards-Dinger and Dieterich 2012), which uses 3D boundary elements to discretize the fault surface, is convenient to employ to generate synthetic earthquake catalogs in a fault system. The central features of this model consist of the use of event-driven 
Table 1 Summary of RSQSim computational algorithm and key features

\begin{tabular}{|c|c|c|}
\hline Steps & Method & Example/Note \\
\hline $\begin{array}{l}\text { 1. Model setup: } \\
\text { define the system } \\
\text { geometry and } \\
\text { initial conditions, } \\
\text { precompute } \\
\text { interaction } \\
\text { matrices, calculate } \\
\text { loading conditions }\end{array}$ & $\begin{array}{l}\text { Interactions } \\
\text { calculated via an } \\
\text { elastostatic } \\
\text { boundary element } \\
\text { method (Okada } \\
\text { 1992). Stress } \\
\text { loading conditions } \\
\text { via backslip }\end{array}$ & $\begin{array}{l}\text { Read in input file and } \\
\text { set up the initial } \\
\text { conditions in the } \\
\text { simulator for a fault } \\
\text { system such as San } \\
\text { Andreas fault } \\
\text { system }\end{array}$ \\
\hline $\begin{array}{l}\text { 2. Determine time of } \\
\text { next sliding state } \\
\text { transition for every } \\
\text { fault element }\end{array}$ & $\begin{array}{l}\text { Analytic } \\
\text { approximations to } \\
\text { evolution of state, } \\
\text { slip speed, and } \\
\text { stress (Dieterich } \\
\text { 1981, 2007) }\end{array}$ & $\begin{array}{l}\text { Very fast due to use } \\
\text { of analytic } \\
\text { approximations. } \\
\text { Scales very well to } \\
\text { many cores }\end{array}$ \\
\hline $\begin{array}{l}\text { 3. Find next system- } \\
\text { wide transition } \\
\text { time: the minimum } \\
\text { of all the times } \\
\text { from step } 2\end{array}$ & $\begin{array}{l}\text { Currently } \\
\text { MPI_Allreduce }\end{array}$ & $\begin{array}{l}\text { This step consumes } \\
\text { much time, } \\
\text { dependent on the } \\
\text { platform } \\
\text { performance }\end{array}$ \\
\hline $\begin{array}{l}\text { 4. Evolve each fault } \\
\text { element to the } \\
\text { transition time } \\
\text { from step } 3\end{array}$ & $\begin{array}{l}\text { Same analytic } \\
\text { expressions used in } \\
\text { step } 2\end{array}$ & $\begin{array}{l}\text { As with step } 2 \text {, very } \\
\text { fast and scales well } \\
\text { to many cores }\end{array}$ \\
\hline $\begin{array}{l}\text { 5. Transition the fault } \\
\text { element whose } \\
\text { time was found in } \\
\text { step } 3 \text { to its new } \\
\text { sliding state. Loop } \\
\text { to step } 2\end{array}$ & $\begin{array}{l}\text { If sliding state } \\
\text { change involves a } \\
\text { change in slip } \\
\text { speed, use } \\
\text { interaction matrices } \\
\text { to update stressing } \\
\text { rates on all other } \\
\text { fault elements }\end{array}$ & $\begin{array}{l}\text { For a state } 0 \text { to state } \\
1 \text { transition, no } \\
\text { system-wide update } \\
\text { is needed. Scales } \\
\text { well to many cores }\end{array}$ \\
\hline
\end{tabular}

computational steps as opposed to time stepping at closed spaced intervals to speed up and the use of analytical approximations in determining the state transition times. The RSQSim simulator is implemented for parallel computations on varying scales, and Table 1 shows a summary of the computational algorithm, key features, and performance analysis.

The simulator code has been benchmarked against a fully dynamic finite element calculation that takes a completely different calculation approach, and the agreement between the two codes is found to be quite satisfactory (Dieterich and Richards-Dinger 2010; Richards-Dinger and Dieterich 2012).

From the perspective of large-scale computations, since the earthquake simulator uses a large number of elements to represent the fault surface, the size of the interaction matrix is proportional to the square of the number of the fault elements, (Eq. 2) which might pose a problem for a large-scale calculation even on a supercomputer with hundreds of thousands of processors. In the parallel implementation, the memory per MPI process is approximately expressed as follows:

$584 N+\frac{16 N^{2}}{n_{\mathrm{p}}}+8 n_{\mathrm{p}}+40 n_{\mathrm{Eq}}$,

where $N$ is the number of elements, $n_{\mathrm{p}}$ is the number of MPI processes, and $n_{\mathrm{Eq}}$ is the number of earthquakes desired. The first term defines the properties for all the elements needed to update in each MPI process, and the second represents the interaction matrix calculation (Eq. 2). While $N$ and np are both large, the first term would become comparable relative to the second term (which is dominant when $N$ and $n_{\mathrm{p}}$ small). In such cases, we use the OPENMP to reduce the number of MPI processors and use multiple threads to reduce the memory requirement; therefore a hybrid method is essential in very large-scale simulator calculations. Using MPI_Allreduce (Table 1) for communications is very expensive in terms of the time spent when a large number of cores are involved. Therefore, the additional benefit using the hybrid method is to greatly reduce the intra-process communications to speed up.

\subsection{Rate-and-state seismicity rate calculation}

We use the seismicity rate formulation of Dieterich (1994) to simulate aftershock rates at a receiver point (cell center) after the synthetic earthquakes are generated. The formulation employs the Coulomb stress change due to a shock and a state variable evolving with time:

$R=\frac{r}{\dot{S}_{r} \gamma}$

where $R$ is the seismicity rate, $r$ is steady-state background rate, $\dot{S}_{r}$ is background Coulomb stressing rate, $\gamma$ is the state variable which evolves with time and Coulomb stress:

$\mathrm{d} \gamma=\frac{[\mathrm{d} t-\gamma \mathrm{d} S]}{(a \sigma)}$,

where $S$ is Coulomb stress on a plane of interest, $a$ is the model constant, $\sigma$ is regional normal stress. If an earthquake strikes, it activates an instantaneous Coulomb stress step $\Delta S^{\mathrm{E}}$ and transfers the state variable from $\gamma_{n}$ to $\gamma_{n+1}$ by:

$\gamma_{n+1}=\gamma_{n} \exp \left(\frac{-\Delta S^{\mathrm{E}}}{a \sigma}\right)$

It is seen that the seismicity rate $R$ jumps with a drop of $\gamma\left(\Delta S^{\mathrm{E}}>0\right.$ and drops with an increase of $\gamma\left(\Delta S^{\mathrm{E}}<0\right.$, consistent with the empirical observations (e.g. Harris and Simpson 1998; Durand et al. 2013). 
To calculate off-fault seismicity in a region, the possible failure planes/slip directions at each grid point are defined using the optimal failure planes (King et al. 1994). The induced stresses at each point are resolved on the plane so the Coulomb stress change is computed. Successively, the seismicity rate $R$ at each grid point could be calculated for all the earthquakes in the fault system. Between the two consecutive earthquake times, the seismicity rate is represented by the rate-state formulation (Dieterich 1994; Harris and Simpson 1998; Ziv 2003; Toda et al. 2005):

$R=\frac{r}{\left[\gamma_{n} \dot{S}_{r} \exp \left(-\frac{\Delta S^{\mathrm{E}}}{a \sigma}\right)-1\right] \exp \left(-\frac{t \dot{S}_{r}}{a \sigma}\right)+1}$,

where $r$ is the reference rate of earthquakes, $t$ is time from the main shock, $\dot{S}_{r}$ is long-term stressing rate. The seismicity rate can increase or decrease with time, depending on the stress transfer due to the main shock and evolution of the state variable $\gamma$.

In creating the synthetic aftershock sequences, we follow the steps given by Smith and Dieterich (2010), assuming that the aftershocks at each grid point follow a random non-stationary Poisson process. By using the seismicity rate $R$ (Eq. 7) as the earthquake occurrence rate at each grid point in a region, we draw the earthquake occurrence times from this rate distribution to generate an aftershock sequence at a grid point. Eventually, we merge and sort all the synthetic aftershock sequences from each grid point into a regional catalog for analysis of the aftershock distribution characteristics in this region.

\section{Examples}

3.1 Synthetic earthquakes in the ALLCAL2 fault system

As an example, we use the rate-and-state RSQSim simulator to calculate the synthetic earthquakes in the ALLCAL2 fault model for the main faults in California (Field et al. 2009; Tullis et al. 2012). Figure 1 shows the locations of the ALLCAL2 faults. The fault surfaces are discretized into square elements of $3 \mathrm{~km} \times 3 \mathrm{~km}$ and involve $\sim 15,000$ fault elements. The depth of faults extends down to $12 \mathrm{~km}$. The slip rate and direction of each element are both specified by the fault model. The uniform constitutive properties used in the RSQSim simulator are $a=0.01, b=0.015, m_{0}=0.6$; these are typical laboratory values (Dieterich and RichardsDinger 2010). The simulator run has a simulation of 410,000 earthquakes spanning $\sim 64,000$ years. Figure 2 shows that most of the earthquakes are between magnitudes of 5-6 due

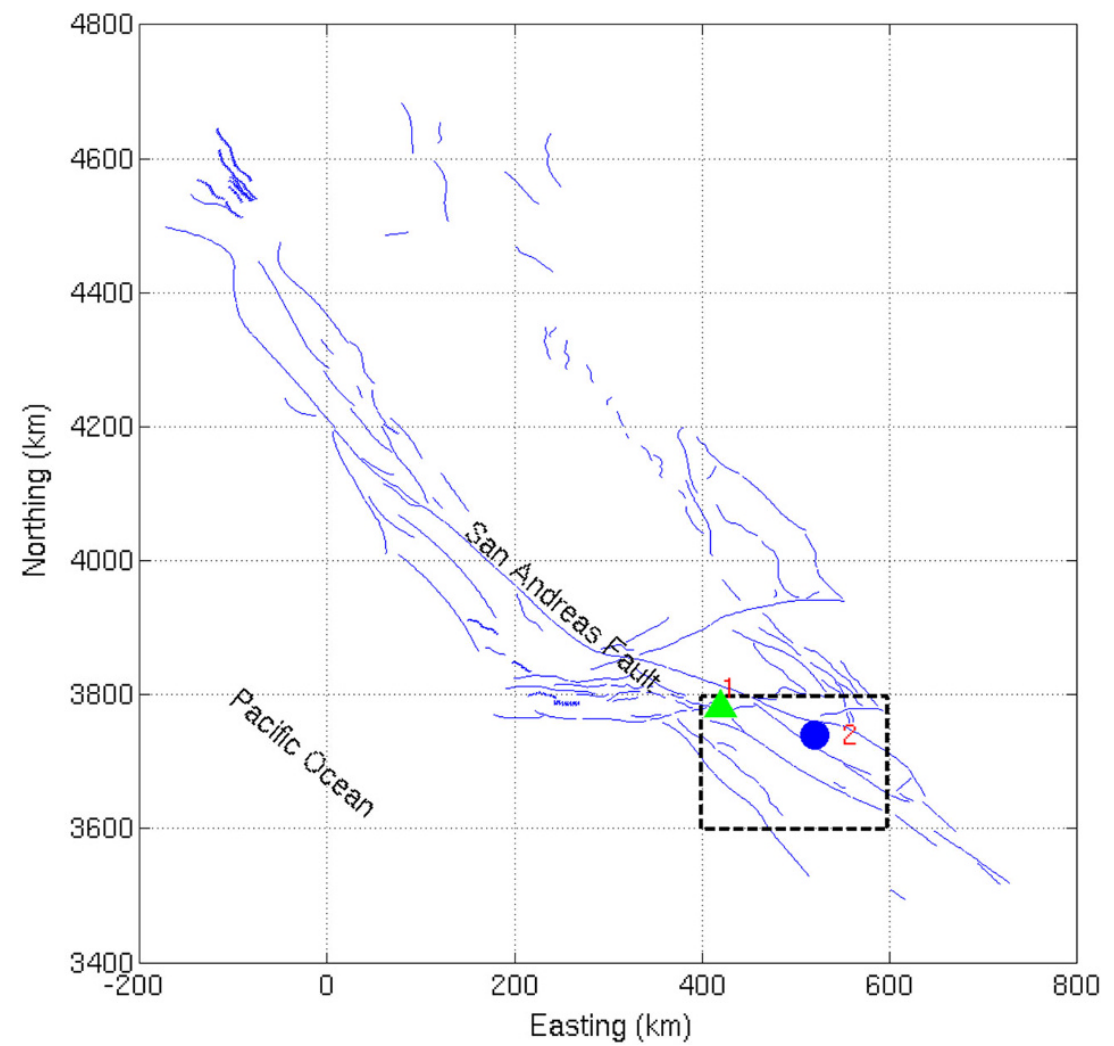

Fig. 1 Fault locations (blue traces) in the ALLCAL2 fault model. The box indicates the area for seismicity calculations later. Two surface locations are marked as location 1 and 2 for later seismicity analysis 


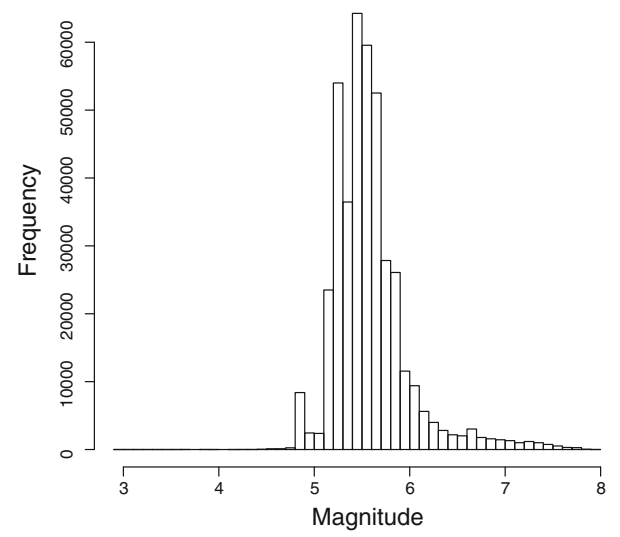

Fig. 2 Earthquake magnitude-frequency histogram in the synthetic catalog. The dominant magnitudes are between 5 and 6 due to the large size of fault elements

to the size of the fault elements. Figure 3 shows an example coseismic rupture extent due to an earthquake on the Anza fault. It has a magnitude of $M=7.5$, extends about $100 \mathrm{~km}$, and lasts about $28 \mathrm{~s}$ with a maximum slip of $5.332 \mathrm{~m}$. The red region indicates the hypocenter. The surrounding fault elements are also shown together and it illustrates the complex fault structure such as fault crossing and gapping. The big earthquake prior to this (3.5 years ago) occurred in Parkfield with a magnitude of $M 7.1$ and the next big one (8.65 years) occurred in southern end of the San Andreas Fault with a magnitude of M7.28. Both are not shown here.

\subsection{Calculation of seismicity}

Using the synthetic earthquake catalog in the ALLCAL2 fault system obtained from above, we calculate the seismicity rate on surface in a region of $200 \mathrm{~km} \times 200 \mathrm{~km}$ in southern
California (box in Fig. 1). The region is divided into $100 \times 100$ cells so each cell is of size $2 \mathrm{~km} \times 2 \mathrm{~km}$. At each grid point (cell center), the optimally oriented fault planes are determined using the sum of two stress rate tensors of (1) the stress rate field generated by the plate tectonic rate $49 \mathrm{~mm} / \mathrm{a}$ (Argus and Gordon 2001) in the direction of $\mathrm{N} 38.6^{\circ} \mathrm{W}$ and (2) the stress rate field generated by the long-term loading rate on the fault surface from the ALLCAL2 model. This approach is different from using the sum of the regional stress fiseld and coseismic stress field (e.g. King et al. 1994), and the optimalorientated planes stay fixed since we aim to understand the long-term seismic hazard characteristics in this region of interest. In this region, the focal mechanism is of essentially strike-slip type, and the right-lateral fault orientation is selected in this study to be consistent with the dominant fault orientations in the fault system. The stressing rate $\dot{S}_{r}$ is calculated by resolving the plate tectonic rate tensor onto the OOP at each cell center. The static stress transfer due to each earthquake in the catalog is conveniently calculated using the dislocation theory in the uniform half-space (Okada 1992) and resolved onto the determined optimal fault plane. All the cells are presumably independent thus no stress coupling between cells is involved in this study.

The seismicity rate at each grid point is calculated by Eq. 7 with $a \sigma=0.16 \mathrm{MPa}$ for each earthquake time. The homogeneous half-space has the elastic constants of $\lambda=\mu=30 \mathrm{GPa}$ in the stress transfer calculations (Okada 1992). Figure 4 shows examples of seismicity rate (normalized by $r$ ) after two main events at two locations labeled $\# 1$ and \#2 in the southern extent of the San Andreas Fault (Fig. 1). The two events used occurred in the San Bernardino area. The blue curves denote the calculated seismicity rates by Eqs. 6 and 7. The red asterisks represent the seismicity

\footnotetext{
Event \# 205003; $M=7.5 ; d t-=-27.5$ secs; $d t=28 ; d t+=-27.5$ secs

Origin time (yrs): 31775.583 Nucleated on patch 1957 (Anza) $\max$ slip $=5.332 \mathrm{~m}$ full color scale slip $=5.3$ :
}

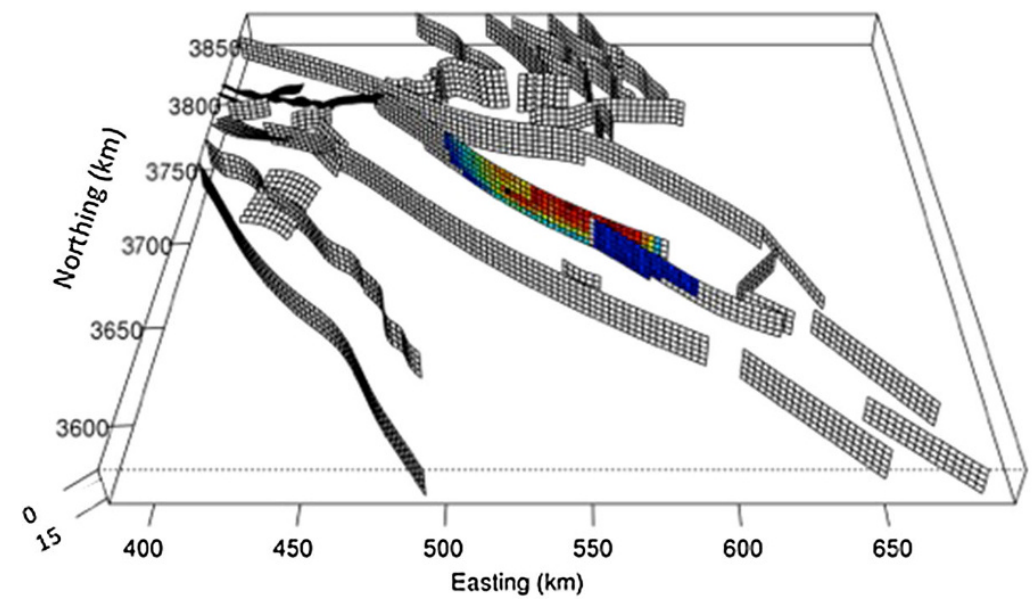

Fig. 3 Rupture extent (color portion) of the earthquake M7.5 on the Anza fault. The maximum slip is about $5.33 \mathrm{~m}$. The surrounding faults are also plotted 

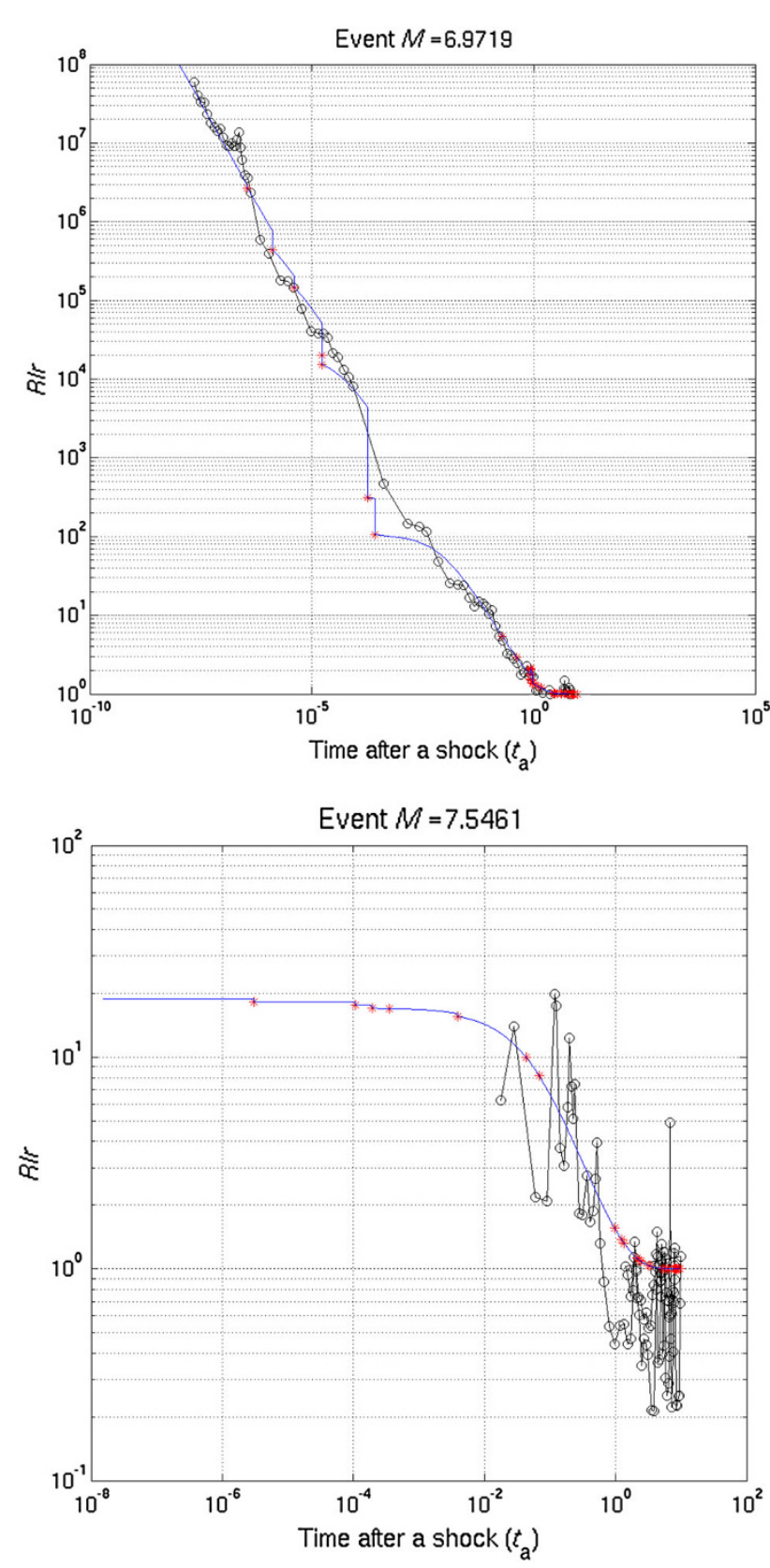

Fig. 4 Seismicity rates (normalized) after main shocks at two locations. Top location 1 after an earthquake M6.9, stress transfer is 17.5 MPa. Bottom location 2 after an earthquake M7.5. The stress transfer is $0.4 \mathrm{MPa}$. Both earthquakes occur in the San Bernardino region. The blue lines indicate the seismicity rate calculated from Eq. 7 and the red stars denote the fault earthquake times. The decay rates at the two locations appear different due to the difference on the stress transfer magnitudes. The circles denote the calculated seismicity rate by drawing the earthquake occurrence times with the seismicity rate (blue lines)

rates at the synthetic earthquake (RSQSim earthquakes) times from the main shock. Note that the seismicity rates jump vertically by the Coulomb stress transfer due to the main shocks (Eq. 6). In this study, only earthquakes greater than M5.5 are considered in seismicity calculations and the

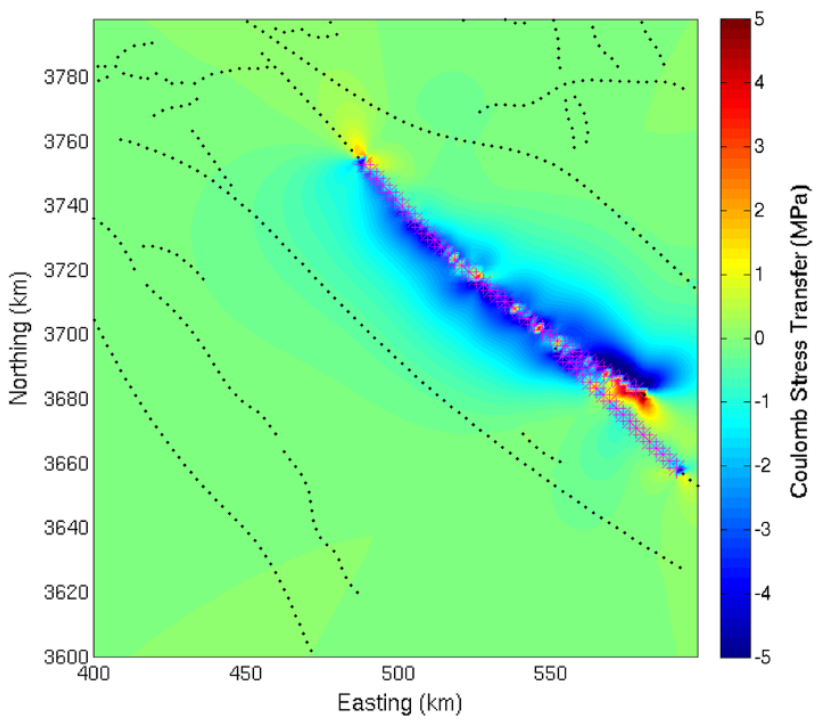

Fig. 5 Coulomb stress map after an earthquake $M 7.5$ on the Anza fault. The red stars indicate the rupture fault region, and the black dots indicate the fault traces in ALLCAL2 fault model. Greater Coulomb stress transfers occur near the ruptured area

reference seismicity $r=2$ per year per cell. The stressing rates are $0.066 \mathrm{MPa}$ per year and $0.058 \mathrm{MPa}$ per year at location 1 and 2, and the characteristic relaxation time for the perturbation of earthquake rate (Dieterich 1994) $t_{a}=a \sigma / \dot{S}_{r}=2.4$ and 2.7 years, respectively. The stressing rate $\dot{S}_{r}$ is calculated by resolving the plate tectonic rate tensor onto the OOP. Both curves at the two locations show decay with time after the main shock and eventually flatten out at the reference level. Note that location 1 is in an active area while location 2 is quiet as shown by the seismicity rate. It is seen that the decay at station 1 is faster by a factor of $10^{8}$ but at station 2 slower by a factor of 20 only. The difference between the decay rates at stations 1 and 2 is because the immediate decay after the main shock is dependent on the stress transfer (Eq. 7), that is 17.5 and $0.48 \mathrm{MPa}$ at location 1 and 2, respectively, and the subsequent rates depend the time elapse and contributions from other earthquakes though these cause much less stress transfer relative to the main shocks at the two locations in this study. The circles Fig. 4 represent the seismicity rates calculated by using the derived seismicity rate (solid line, from Eq. 7) and using the nonstationary Poisson process to draw the earthquake occurrence times for the calculations below. The two curves are in close proximity and the agreement is satisfactory.

For the region marked as a box in Fig. 1, the Coulomb stress changes in Fig. 5 are calculated after the main shock of M7.5 (Fig. 3). The dotted lines indicate the fault traces in this region, and the red stars denote the rupture extent (as in Fig. 3). The Coulomb stress transfer appears to be concentrated near the rupture with positives on both ends and on a few spots along the rupture region. It is noted that the 

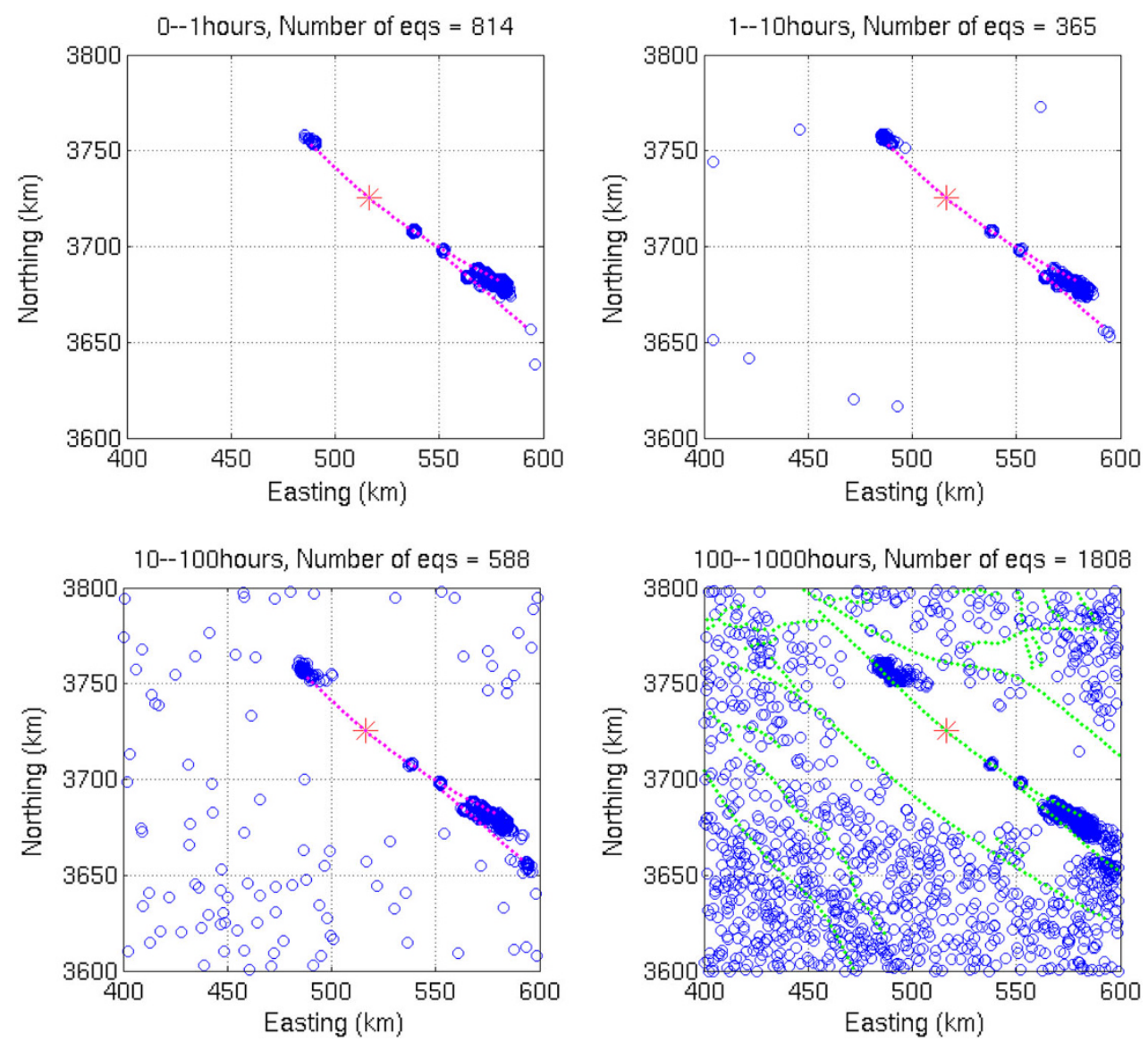

Fig. 6 Aftershock (circles) distribution after a main shock of M7.5. It is seen immediately after the main shock, the seismicity rate is elevated near the ruptured fault (red dashed lines) and then migrates outward. Stress shadow is seen by the rupture (Fig. 5). Green lines are fault traces and the red star indicates the nucleation point

negative Coulomb stress change extends to the adjacent faults and covers more area than the positive stress transfer. The range of the stress change is around -5 to $5 \mathrm{MPa}$, so using the stressing rate $\sim 0.06 \mathrm{MPa} /$ year, the main shock M7.5 could advance or delay the earthquake clock by $\sim 80$ years in the rupture region. The distribution of aftershocks in a few intervals from the main shock is estimated using the sampling samples based on the seismicity rate and is shown in Fig. 6. The intervals are 0-1, 1-10, 10-100 and 100-1,000 $\mathrm{h}$ ( 4-40 days) after the main shock. The circles indicate the possible earthquake locations, the red-dashed lines denote the rupture extent, the red star denotes the nucleation location of the main shock, and the green-dashed lines indicate the location of the faults in this region. It is clearly seen that immediately after the main shock the aftershocks are concentrated in the vicinity of the ruptures, and then migrate with time away from the ruptures. Eventually, the distribution moves toward the background seismicity distribution although it might take different times in different areas, depending on the Coulomb stress transfer and stressing rate. This is similar to the seismicity pattern calculated from a uniform slip in a 3D heterogeneous stress field (Smith and Dieterich 2010). Note that due to the negative Coulomb stress transfer by the rupture (Fig. 5), that intends to push a fault farther from failure, the seismicity rate in this region may take tens of years to recover the significantly depressed seismicity rate to the background rate level.

Seismicity rates as a function of time near the rupture region (so the background seismicity is most excluded) are shown in Fig. 7. It is seen that the rate curve falls as about $t^{-0.9}$ nearly until a few days after the main shock and then the slope starts to decrease due to the effect of the background seismicity, approximately following the Omori decay law. The decay rate is consistent with those found from uniform static stress change or from a uniform slip on finite faults in a 3D heterogeneous stress field (Smith and Dieterich 2010) and is also close to that calculated for Landers aftershock activity using the rate-state model (Hainzl et al. 2009). The result demonstrates that the rate-state formulation not only replicates the Omori aftershock (synthetic earthquakes on faults) decay rate (Dieterich and Richards-Dinger 2010), but also suggests earthquake probabilities in a region might have a similar decay law as well, as seen in this study (without stress coupling between different cells). 


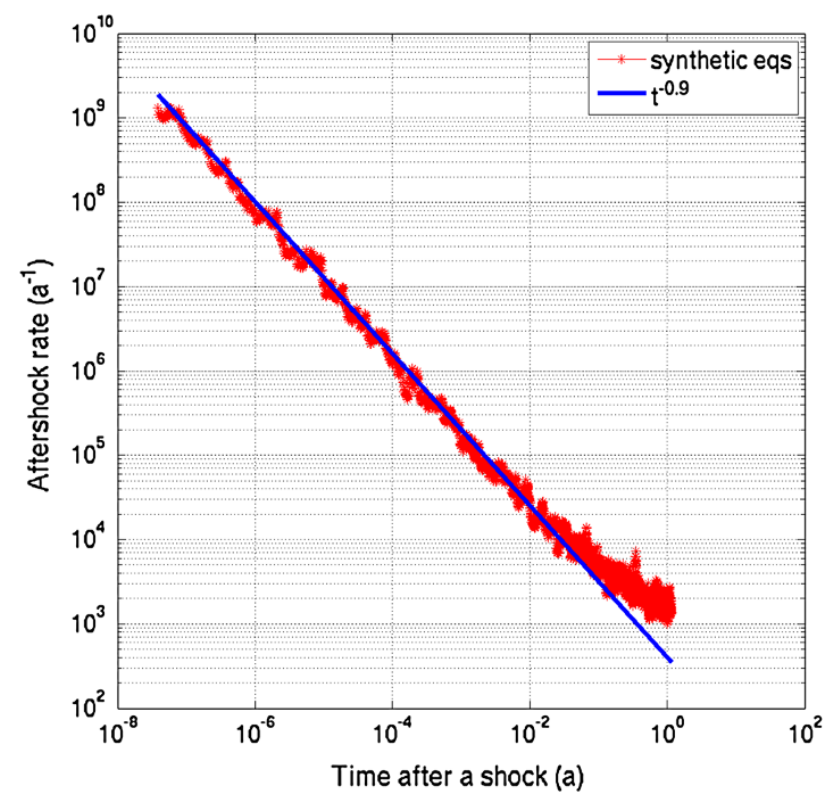

Fig. 7 Decay of aftershock rate with time from the mainshock M7.5. The slope is 0.9 for the blue line. It is seen the aftershock rate appears to begin to move up at $\sim 30$ days after depressed by the negative stress transfer (Fig. 5)

\section{Discussion and conclusions}

We employ the efficient rate-and-state earthquake simulator RSQSim to generate synthetic earthquakes in the fault system (ALLCAL2) and calculate the earthquake probabilities in a region from the synthetic earthquakes. The effect of the earthquakes in the fault model on the off-fault seismicity is obtained by calculating the Coulomb stress transfer on the optimal-oriented planes. The rate-state seismicity equation relates the Coulomb stress transfer to the seismicity rate change. The factors controlling the seismicity rate include the stressing rate, Coulomb stress transfer as well as the background rate that is constant in this study. The possible earthquake occurrence in a region is estimated using the seismicity rate as non-stationary Poisson rate. The results show that the aftershocks immediately after a main shock are concentrated near the rupture area, and then disperse into the surrounding area to reach uniformity eventually within a variable time frame. The total number of aftershocks near the rupture region decay with time, also resembling the Omori decay law, with a power exponent of 0.9 similar to the decay obtained for the uniform slip on finite faults (Smith and Dieterich 2010). The decay rate is close to the decay rates found for some big earthquakes in the region (Toda et al. 2005; Hainzl et al. 2009). The example results strongly demonstrate that the rate-and-state fault system earthquake simulator and the seismicity equations that are based on the rate-state friction nucleation of earthquake are well posited to characterize the aftershock distribution in regional assessments of earthquake probabilities.
The method described above to calculate the off-fault seismicity in a region is somewhat similar to that used in the stress release model of statistical earthquake occurrence (Keuhn et al. 2008) and to that used in the ETAS model (e.g. Bansal and Ogata 2013) but the main difference is that the stress in the stress release model is only a symbolic scalar related to the magnitude and not the true stress tensor as used in the rate-state seismicity rate model that can be connected with a physics-based earthquake simulator like RSQSim to better model the entire fault system and off-fault regions, and the ESTAS model uses the spatially distributed four model constants for seismicity rates. The background seismicity is simply set constant in this study but might be time-dependent as suggested by the ETAS model results in optimal estimates from hypocenter data in some regions (e. g. Bansal and Ogata 2013; Kumazawa and Ogata 2013). The same calculation procedure described above also applies to the scenarios with non-stationary background seismicity to investigate the phenomena such as seismic activation and quiescence but how to determine the time dependence will be a future effort.

It need to be pointed out that in generating the aftershocks in a region, their magnitudes or rupture characteristics cannot be directly derived from the stress transfer model and rate-state seismicity equations. The simulation of the magnitude distribution may be carried out using the Gutenberg-Richter distribution (e.g. Keuhn et al. 2008; Gu et al. 2013) to complete the earthquake probability estimation. Other additions, such as realistic background seismicity rate distribution, stress-interactions between adjacent regions instead of assuming the independence among regions, and dynamic stress transfer, would help improve evaluations of seismic hazard in a region (e.g. Kilb et al. 2002; Keuhn et al. 2008; Durand et al. 2013).

Acknowledgments This research is supported by the NSF Frontiers in Earth-System Dynamics (EAR-1135455). The computational work used the Extreme Science and Engineering Discovery Environment (XSEDE), which is supported by National Natural Science Foundation grant No. OCI-1053575. This research is part of the Blue Waters sustained-petascale computing project, which is supported by the National Natural Science Foundation (award No. OCI 07-25070) and the state of Illinois. Blue Waters is a joint effort of the University of Illinois at Urbana-Champaign and its National Center for Supercomputing Applications. We are grateful to the anonymous reviewers for critical suggestions and comments which have greatly improved the manuscript.

\section{References}

Argus DF, Gordon RG (2001) Present tectonic motion across the Coast Ranges and San Andreas fault system in central California. GSA Bull 113(12):1580-1592

Bansal AR, Ogata Y (2013) A non-stationary epidemic type aftershock sequence model for seismicity prior to the December 26, 2004 M9.1 Sumatra-Andaman islands mega-earthquake. J Geophys Res 118:616-629. doi:10.1002/jgrb.50068 
Dieterich JH (1979) Modeling of rock friction, 1. Experimental results and constitutive equations. J Geophys Res 84(B5):2161-2168

Dieterich JH (1981) Constitutive properties of faults with simulated gauge. In: NL Carter et al (ed) Mechanical behavior of crustal rocks: The handin volume. Geophysical Monograph Series, vol 24. AGU, Washington, D.C, pp 103-120

Dieterich JH (1994) A constitutive law for rate of earthquake production and its application to earthquake clustering. J Geophys Res 99(B2):2601-2618

Dieterich JH (1995) Earthquake simulations with time-dependent nucleation and long-range interaction. Nonlinear Processes Geophys 2:109-120

Dieterich JH (2007) Applications of rate- and state-dependent friction to models of fault slip and earthquake occurrence. In: $\mathrm{H}$ Kanamori (ed) Treatise on geophysics. Earthquake Seismology, vol 4, Elsevier, Boston, pp 107-129

Dieterich JH, Richards-Dinger KB (2010) Earthquake recurrence in simulated fault systems. Pure Appl Geophys 167:1087-1104

Durand V, Bouchon M, Karabulut H, Marsan D, Schmittbuhl J (2013) Link between Coulomb stress changes and seismic activation in the eastern Marmara sea after the 1999, Izmit (Turkey), earthquake. J Geophys Res 118:681-688. doi:10.1002/jgrb. 50077

Field EH, Dawson TE, Felzer KR, Frankel AD, Gupta V, Jordan TH, Parsons T, Petersen MD, Stein RS, Weldon RJ II, Wills CJ (2009) Uniform California earthquake rupture forecast, version 2 (UCERF 2). Bull Seismol Soc Am 99:2053-2107. doi:10.1785/ 0120080049

Gomberg JN, Beeler M, Blanpied ML, Bodin P (1998) Earthquake triggering by transient and static deformation. J Geophys Res 103(B10):24411-24426

Gomberg JN, Beeler N, Blanpied M (2000) On rate-state and Coulomb failure models. J Geophys Res 105:7857-7871

Gross S, Burgmann R (1998) Rate and state of background stress estimated from the aftershocks of the 1989 Loma Prieta, California, earthquake. J Geophys Res 103:4915-4927

Gu C, Schumann AY, Baiesi M, Davidsen J (2013) Triggering cascades and statistical properties of aftershocks. J Geophys Res 118:4278-4295. doi:10.1002/jgrb.50306

Hainzl S, Enescu B, Cocco M, Woessner J, Catalli F, Wang R, Roth F (2009) Aftershock modeling based on uncertain stress calculations. J Geophys Res 114:B5. doi:10.1029/2008JB006011

Harris RA (1998) Introduction to special section: stress triggers, stress shadows and implications for seismic hazard. J Geophys Res 103(B10):24347-24358

Harris RA, Simpson RW (1998) Suppression of large earthquakes by stress shadows: a comparison of Coulomb and rate-and-state failure. J Geophys Res 103(B10):24439-24451

Keuhn NM, Hainzel S, Scherbaum F (2008) Non-Poissonian earthquake occurrence in coupled stress release models and its effect on seismic hazard. Geophys J Int 174:649-658

Kilb D, Gomberg J, Bodin P (2002) Aftershock triggering by complete Coulomb stress changes. J Geophys Res 107:B4. doi:10.1029/2001JB000202

King GCP, Stein RS, Lin J (1994) Static stress changes and the triggering of earthquakes. Bull Seismol Soc Am 84(3):935-953

Kumazawa T, Ogata Y (2013) Quantitative description of induced seismic activity before and after the 2011 Tohoku-Oki earthquake by nonstationary ETAS models. J Geophys Res 118:6165-6182. doi:10.1002/2013JB010259

McCloskey J, Nalbant SS, Steacy S, Nostro C, Scotti O, Baumont D (2003) Structural constraints on the spatial distribution of aftershocks. Geophys Res Lett 30(12):1610. doi:10.1029/ 2003GL017225

Ogata Y (1988) Statistical models for earthquake occurrences and residual analysis for point processes. J Am Stat Assoc 83:9-27

Ogata Y, Jones LM, Toda S (2003) When and where the aftershock activity was depressed: Contrasting decay patterns of the proximate large earthquakes in southern California. J Geophys Res 108(B6):2318. doi:10.1029/2002JB002009

Okada Y (1992) Internal deformation due to shear and tensile faults in a halfspace. Bull Seismol Soc Am 82:1018-1040

Richards-Dinger K, Dieterich JH (2012) RSQSim earthquake simulator. Seismol Res Lett 83:985-990

Ruina AL (1983) Slip instability and state variable friction laws. J Geophys Res 88:10359-10370

Smith DE, Dieterich JH (2010) Aftershock sequence modeled with 3D stress heterogeneity and rate-state seismicity equations: implications for crust stress estimation. Pure appl Geophys 167:1067-1085

Steacy S, Goomberg J, Cocco M (2005) Introduction to special section: stress transfer, earthquake triggering and time-dependent seismic hazard. J Geophys Res 110:B05S01. doi:10.1029/ 2005JB003692

Steacy S, Nalbant SS, McCloskey J (2005) Onto what planes should Coulomb stress perturbations be resolved? J Geophys Res 110:B05S15. doi:10.1029/2004JB003356

Toda SS, Stein S, Reasenberg PA, Dieterich JH (1998) Stress transferred by the $M_{\mathrm{w}}=6.5$ Kobe, Japan shock effect on aftershocks and future earthquake probabilities. J Geophys Res 103:24543-24565

Toda S, Stein RS, Richards-Dinger K, Bozkurt SB (2005) Forecasting the evolution of seismicity in southern California: animations built on earthquake stress transfer. J Geophys Res 110:B05S16. doi: 10.1029/2004JB003415

Tullis TE, Richards-Dinger K, Barall M, Dieterich JH, Field EH, Heien EM, Kellogg LH, Pollitz FF, Rundle JB, Sachs MK, Turcotte DL, Ward SN, Yikilmaz MB (2012) A Comparison among observations and earthquake simulator results for the ALLCAL2 California fault model. Seismol Res Lett 83:6. doi:10.1785/0220120094

Vere-Jones D (1978) Earthquake prediction-a statistician's view. J Phys Earth 26:129-146

Xu C, Wang J, Li Z, Drummond J (2010) Applying the Coulomb failure function with an optimally orientated plane to the 2008 $M_{\mathrm{w}} 7.9$ Wenchuan earthquake triggering. Tectonophysics 491:119-126

Ziv A (2003) Foreshocks, aftershocks and remote triggering in quasistatic fault models. J Geophys Res 108(B10):2498. doi:10.1029/ 2002JB002318

Ziv A, Rubin AM (2003) Implications of rate-and-state friction for properties of aftershock sequence: quasi-static inherently discrete simulations. J Geophys Res 108(B1):2051. doi:10.1029/ 2001JB001219 